\title{
1 A sarbecovirus found in Russian bats uses human ACE2
}

2 Stephanie N. Seifert ${ }^{1}$ and Michael C. Letko ${ }^{1 \#}$

3

4

40 Paul G. Allen School for Global Health

41 Washington State University

42 PO Box 647090

43 Pullman, WA 99164-7090

44 Email: michael.letko@wsu.edu 


\section{ABSTRACT}

46 Spillover of sarbecoviruses from animals to humans resulted in outbreaks of severe acute

47 respiratory syndrome SARS-CoVs and the ongoing COVID-19 pandemic. Efforts to

48 identify the origins of SARS-CoV-1 and -2 has resulted in the discovery of numerous

49 animal sarbecoviruses - the majority of which are only distantly related to known human

50 pathogens and do not infect human cells. The receptor binding domain (RBD) on

51 sarbecoviruses engages receptor molecules on the host cell and mediates cell invasion.

52 Here, we tested the receptor tropism for RBDs from two sarbecoviruses found in Russian

53 horseshoe bats to screen cell entry. While these two viruses are in a viral lineage distinct

54 from SARS-CoV-1 and -2, one virus was capable of using human ACE2 to facilitate cell

55 entry. Our findings demonstrate that sarbecoviruses circulating in wildlife outside of Asia

56 also exhibit compatibility with human ACE2 and should be taken into consideration for

57 future universal sarbecovirus vaccine candidates.

58

59

60

61

62

63

64

65

66

67 
Introduction

Zoonotic spillover of sarbecoviruses from animals to humans has led to the emergence of highly pathogenic human viruses, SARS-CoV-1 and -2 , with the later

71 leading to the largest global pandemic in modern history. Researchers around the world

72 are ramping up the pace of viral discovery efforts, expanding the sequence databases with

73 new animal sarbecoviruses in circulation. While some laboratory experiments have been

74 performed with these new viruses, demonstrating a range of host tropisms, several viruses

75 remain untested, and thus their ability to transmit to humans is unknown.

76 Coronaviruses are covered with a "spike" glycoprotein that engages with receptor

77 molecules on the surface of host cells and mediates viral infection of the cell. A small

78 region within the spike proteins of sarbecoviruses, known as the receptor binding domain

79 (RBD), contains all of the structural information necessary to engage with the host

80 receptor. We and others have experimentally classified the majority of published

81 sarbecovirus RBDs into different clades based on sequence and functional data: clade 1,

82 identified in Asian bats, contains no deletions and binds to host receptor, Angiotensin-

83 Converting Enzyme 2 (ACE2), whereas clade 2, also identified in Asian bats, contains 2

84 deletions and does not use ACE2 and clade 3 viruses, found more widely in Africa and

85 Europe, contain 1 deletion and also infect using an unknown receptor ${ }^{1-3}$. Recently, several

86 viruses were identified in China that comprise a fourth clade that also interact with human

$87 \mathrm{ACE}^{4}$.

In late 2020, two clade 3 sarbecoviruses were identified in Rhinolophus bats in

89 Russia: Khosta-1 was found in Rhinolophus ferrumequinum and Khosta-2 in $R$. 90 hipposideros 5 . Similar to other European and African clade 3 viruses, the Khosta viruses 
91 are divergent from the RBD found in SARS-CoV-1 and -2 , and were thus presumed not to

92 possess any tropism for human cells. However, a recent study demonstrated that a new

93 clade (clade 4) of sarbecoviruses found in China containing a deletion similar to clade 3

94 sarbecoviruses can bind to human ACE2 ${ }^{4}$. Here, we tested the RBDs from Russian

95 sarbecoviruses, along with other clade 3 viruses and report that the virus, Khosta-2, is

96 capable of infecting cells using the human orthologue of ACE2.

\section{Results}

100 Khosta virus receptor binding domains are distinct from human viruses

101 Khosta-1 and -2 were identified by Alkhovsky and colleagues in bat samples 102 collected between March-October 2020 near Sochi National Park ${ }^{5}$. Phylogenetic analysis 103 of the conserved viral gene, Orf1ab, revealed these viruses were most closely related to 104 another sarbecovirus found in Bulgaria in 2008 (known as BM48-31 or Bg08), and form a 105 lineage sarbecoviruses distinct from human pathogens, SARS-CoV-1 and $-2^{5}$. A list of 106 viruses and accession numbers used in this study can be found in table 1 . Phylogenetic 107 analysis of the spike RBD further reflected the close relatedness between Khosta -1 and 1082 with BM48-31 and other clade 3 RBD viruses we have previously tested from Uganda 109 and Rwanda ${ }^{1,6}$ (Fig. 1a). Clade 3 RBDs, including the Khosta viruses, contain a truncated 110 surface-exposed loop, as compared to the ACE2-dependent, clade 1 viruses such as

111 SARS-CoV and additionally vary in most of the residues known for clade 1 viruses to 112 interact with ACE2 $2^{1,2,6,7}$. 
114 RBD from Khosta viruses mediate entry into human cells

SARS-CoV-1 spike with the Khosta RBDs and generated chimeric spike expression

117 plasmids (Fig. 1b) ${ }^{1}$. For comparison, we also included chimeric RBD spikes for other clade

1183 RBDs we have previously tested (BM48-31, Uganda, Rwanda) as well as SARS-CoV-2

119 and related RatG13 viruses. These chimeric spike expression constructs were used to

produce BSL2-compatible viral reporter pseudotypes with Vesicular Stomatitis Virus

121 expressing a dual GFP-luciferase reporter ${ }^{1}$. All of the chimeric spike proteins expressed

122 to similar levels in mammalian cells and incorporated in Vesicular Stomatitis Virus (VSV).

123 Chimeric spike with the RBD from BM48-31 and RatG13 showed reduced incorporation

124 but this did not correlate with viral entry phenotypes observed in later experiments (Fig.

125 1c, e).

To test human cell compatibility, we first infected the human liver cell line, Huh-7,

127 with pseudotypes bearing the chimeric Khosta RBD spikes. In the absence of the addition

128 of an exogenous protease, trypsin, the pseudotypes exhibited almost no entry in these

129 cells, which has been observed for other sarbecoviruses and is attributed to low

130 endogenous expression of ACE2. However, when trypsin was included during the

131 infection, entry signal strongly increased for SARS-CoV-1 and -2 RBDs as well as the

132 Khosta RBDs (Fig. 1d). As we and others have shown previously, trypsin enhancement of

133 sarbecovirus entry is still receptor dependent, suggesting that the Khosta virus RBDs were

134 using a receptor present in human cells to mediate infection ${ }^{1,8}$. 


\section{The RBD from Khosta-2 infects cells using human ACE2}

To characterize potential receptors that the Khosta viruses may be using, we

139 performed a classic receptor tropism test, where we transfected Baby Hamster Kidney

140 (BHK) cells with human orthologues of known coronavirus receptors and then infected with

141 our pseudotype panel. Unlike 293T cells, which express low levels of human ACE2 and

142 potentially other coronavirus receptors and have been shown to have low but measurable

143 permissivity to SARS-CoV infection, BHK cells are generally considered completely non-

144 permissive for sarbecoviruses unless a suitable receptor is supplemented ${ }^{9}$. The Khosta-1

145 RBD failed to infect cells expressing any of the receptors, while Khosta 2 RBD clearly

146 infected cells expressing human ACE2 (figure 1e). The level of cell entry mediated by the

147 Khosta 2 RBD was similar to RatG13, a bat sarbecovirus with high similarity to SARS-

148 CoV-2 in the RBD that also binds human ACE2, albeit with lower efficiency than the human

149 pathogen $^{10,11}$ (Fig. 1e). In contrast to the ACE2 results, only HCoV-229E could infect cells

150 expressing Aminopeptidase N (APN), and MERS-CoV spike could only infect cells

151 expressing dipeptidyl peptidase IV (DPP4) - the known receptors for these viruses,

152 demonstrating these receptors were expressed to functional levels (Fig. 1e).

\section{Discussion}

To date, all animal derived sarbecoviruses capable of infecting cells through

156 human ACE2 have been found in Asia ${ }^{2,12-15}$. Here, we provide evidence that animal-

157 derived sarbcoviruses circulating in wildlife outside of Asia can also possess the capacity

158 to infect human cells and use human ACE2 (Fig. 1). Khosta 1 and 2 viruses are most 159 closely related to other clade 3 RBD viruses, which have been found across a much wider 
160 geographic range than the clade 1 viruses ${ }^{1,6,16,17}$. Thus, our findings show that 161 sarbecovirus host receptor compatibility is not geographically locked and that other

162 sarbecoviruses, which may only be distantly related to human SARS-CoVs, may also 163 stochastically possess the capacity to infect humans. Importantly, as the researchers who 164 initially discovered the Khosta viruses note with their findings: the Khosta bat 165 sarbecoviruses are genetically distinct from human SARS-CoVs in that they lack genetic 166 information encoding for some of the genes thought to antagonize the immune system and 167 contribute to pathogenicity, such as Orf85. Unfortunately, because coronaviruses are 168 known to recombine in co-infected hosts, the recent identification of SARS-CoV-2 spillover 169 from humans back in wildlife populations opens the possibility of new human-compatible 170 sarbecoviruses ${ }^{18-23}$.

171 In the presence of trypsin, both Khosta-1 and -2 RBDs were capable of infecting 172 human cells, with Khosta-1 performing notably stronger than Khosta-2, however in our 173 receptor-specific assay, only Khosta-2 could infect cells expressing human ACE2 without 174 exogenous protease (Fig. 1d, e). We have previously shown that a small number of clade 1752 RBDs, such as As6526, also exhibit protease-mediated, ACE2-independent entry, and 176 similar phenotypes have been described for other bat coronaviruses ${ }^{1,24}$. Because not all 177 of the clade 2 and 3 viruses exhibit this phenotype, these findings collectively suggest that 178 other coronaviruses can infect human cells through a presently unknown receptor. 179 Sarbecoviruses have been shown to co-circulate in their reservoirs, so this variation in 180 receptor usage among closely related viruses may even represent an evolutionary strategy 181 for viral persistence ${ }^{2}$. 
Although the Khosta-2 RBD exhibited clear compatibility with human ACE2,

pseudotype entry was approximately 10-fold less than for SARS-CoV-1 and -2, and was

184 to levels closer to RatG13 RBD (Fig. 1e). The RBD for RatG13, while also compatible with

185 human ACE2, has been shown by other groups to exhibit poor binding to human ACE2 ${ }^{25-}$

18627 . A single point mutation, T403R/K, has been shown to improve RatG13-RBD binding

187 and entry with human ACE2, although this mutation is already present in Khosta-2

$188(\mathrm{~K} 403)^{25}$. We have previously demonstrated that MERS-CoV can rapidly adapt to

189 overcome a semi-permissive receptor, suggesting that limited entry phenotypes are easily

190 overcome by betacoronaviruses ${ }^{28}$. As new sarbecoviruses are discovered in wildlife, there

191 is a propensity in the research community to compare single amino acids based on known

192 human SARS-CoVs as an indicator for compatibility with humans. Our work underscores

193 the challenges in predicting viral function from sequence data alone and sheds light on

194 another group of sarbecoviruses with zoonotic potential. Current universal sarbecovirus

195 vaccines in development include mostly clade 1 viruses and one of the clade 2 viruses but

196 do not include any members from clade $3^{29,30}$. Taken together, our findings with the Khosta

197 viruses show that the sarbecoviruses may pose an even broader geographic threat than

198 previously appreciated.

\section{Methods}

201 Phylogenetic analysis

202 Genbank accession numbers for all sarbecovirus spike sequences used in this study are

203 available in table 1. Amino acid sequences for the receptor binding domain of the spike 204 glycoprotein were aligned using ClustalW multiple sequence alignment with default 
205 parameters. A maximum likelihood phylogenetic tree was inferred with PhyML v. $3.0^{31}$

206 using the 'WAG' matrix +G model of amino acid substitution as selected by Smart Model

207 Selection method with 1000 bootstrap replicates ${ }^{32}$. The final tree was then visualized as a

208 cladogram with FigTree v1.4.4 (https://github.com/rambaut/figtree).

210 Plasmids and sequences

211 Untagged human orthologues of ACE2 (Q9BYF1.2), APN (NP_001141.2), and DPP4

212 (XM_005246371.3) were described previously ${ }^{1}$. Spike sequences from HCoV-229E

213 (AB691763.1), MERS-CoV (JX869059.2), and SARS-CoV-1 (AY278741) were codon-

214 optimized, appended with a carboxy-terminal FLAG tag sequence separated by a flexible

215 poly-glycine linker and cloned into pcDNA3.1+ as previously described ${ }^{1}$. The SARS-CoV-

$2161 \mathrm{RBD}$ was removed with Kpnl and Xhol and replaced with codon-optimized, synthesized

217 RBD fragments as previously described ${ }^{1}$.

219 Cells and pseudotype assay

220 293T, Huh-7 (human liver cells), and BHKs were maintained under standard cell culture

221 conditions in DMEM with L-glutamine, antibiotics, and 10\% Fetal Bovine Serum. Single-

222 cycle, Vesicular Stomatitis Virus (VSV) pseudotype assays were performed as previously

223 described ${ }^{1}$. Briefly, 293T "producer cells" were transfected with spike plasmids or empty

224 vector as a "no spike" control and infected 24-hours later with VSV- $\Delta$ G-luc/GFP particles,

225 generating chimeric-spike pseudotyped particles that were harvested 72 hours post-

226 transfection and stored at $-80^{\circ} \mathrm{C}$. Target cells were plated in 96-well format, and spin-

227 infected in quadruplicate with equivalent volumes of viral pseudotypes at $1200 \times \mathrm{f}$ for 1 
228 hour at $4^{\circ} \mathrm{C}$. Infected cells were incubated for approximately 18-20 hours and luciferase

229 was measured using the Promega BrightGlo luciferase kit following manufacturers'

230 instructions (Promega). Entry signal was normalized to the average signal for the "no

231 spike" control. Plates were measured and analyzed in triplicate. Data are representative

232 of four complete biological replicates.

233

234 Westernblot

235 Viral pseudotypes were concentrated and 293T producer cells were lysed in $1 \%$ SDS and 236 clarified as described previously ${ }^{1}$. Lysates were analyzed on $10 \%$ Bis-Tris PAGE-gel

237 (Thermo Fisher Scientific) and probed for FLAG (Sigma-Aldrich; A8592; 1:10000); GAPDH 238 (Sigma-Aldrich, G8795, 1:10000); and/or VSV-M (Kerafast, 23H12, 1:5000). Signal was 239 detected using SuperSignal West substrate (Thermo-Fisher).

\section{AUTHOR CONTRIBUTIONS STATEMENT}

S.N.S. and M.L. performed experiments and wrote the manuscript. M.L. designed the study, analyzed the data, and assembled the figures.

\section{ADDITIONAL INFORMATION}

The authors declare no competing interests. 


\section{Figures, figure legends and tables}
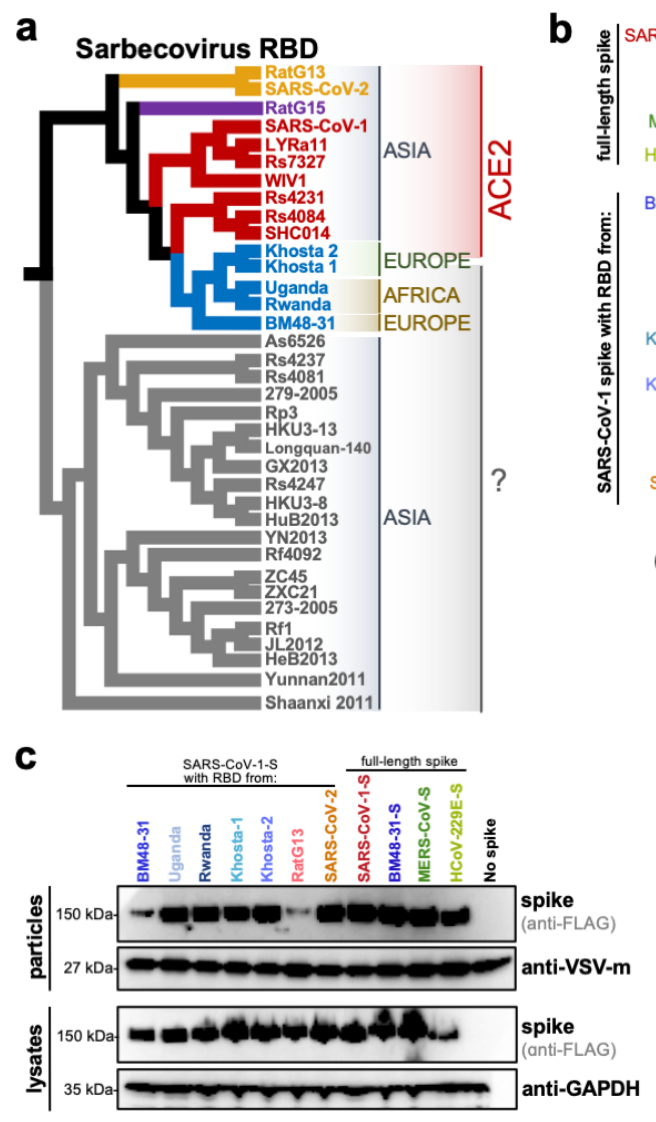

258

259

260

261

262

263

264

265

266

267

268

269

270

271

272

273 b

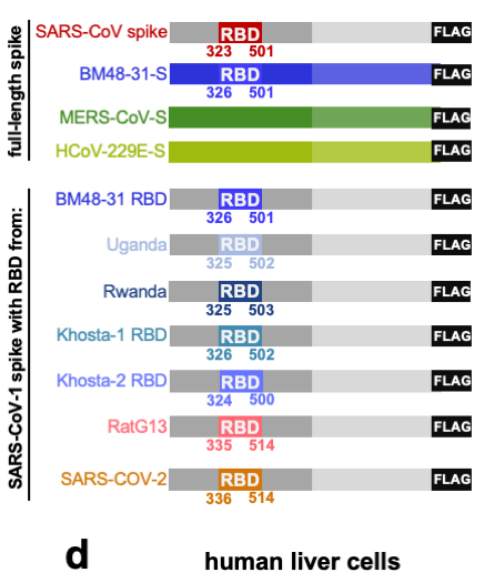

d

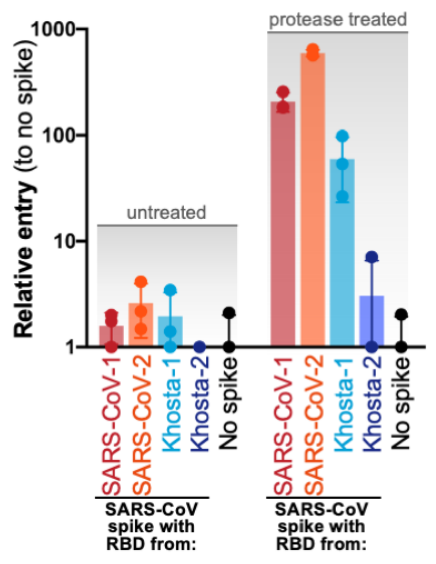

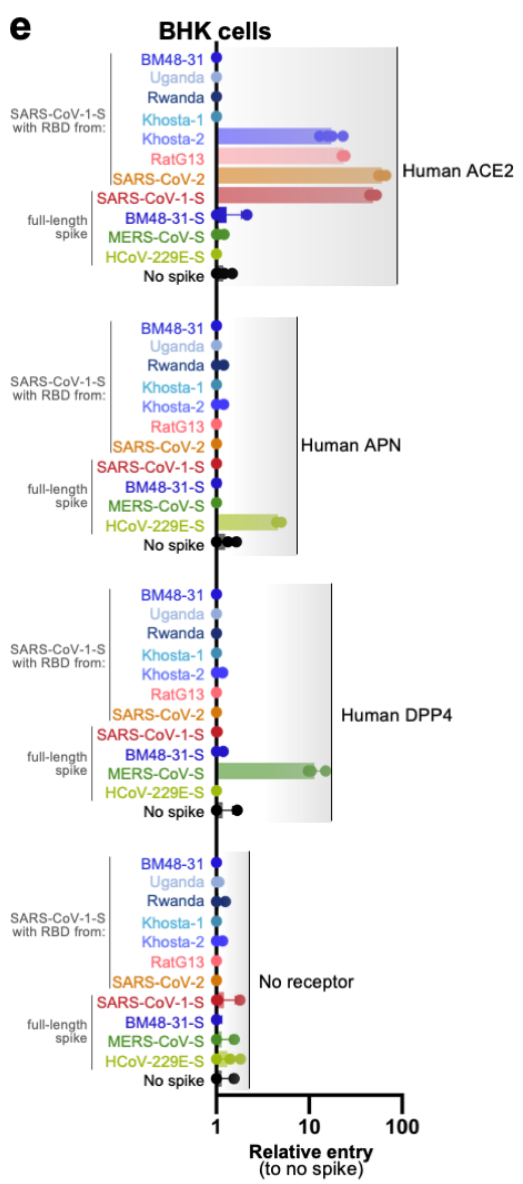

Figure 1. Khosta-2 uses human ACE2 to infect cells. (a) Sarbecovirus Receptor Binding Domain Cladogram based on amino acid sequenced. Countries of origin and known host receptors are indicated to the right. Clade 1 viruses are shown in red and orange, clade 2 in grey, clade 3 in blue and clade 4 in purple. (b) Diagram of spike constructs used for this study. The SARS-CoV-1 RBD was replaced with RBDs from other sarbecoviruses. (c) Expression and incorporation of viral pseudotypes by westernblot. (d) Huh-7 cells were infected with pseudotypes in the presence of absence of trypsin. Cells were infected in triplicate. (e) BHK cells were transfected with receptors and infected in the absence of trypsin. Cells were infected in quadruplicate. 
bioRxiv preprint doi: https://doi.org/10.1101/2021.12.05.471310; this version posted December 7, 2021. The copyright holder for this preprint

(which was not certified by peer review) is the author/funder. All rights reserved. No reuse allowed without permission.

\section{Table 1. Sarbecovirus sequences used in this study}

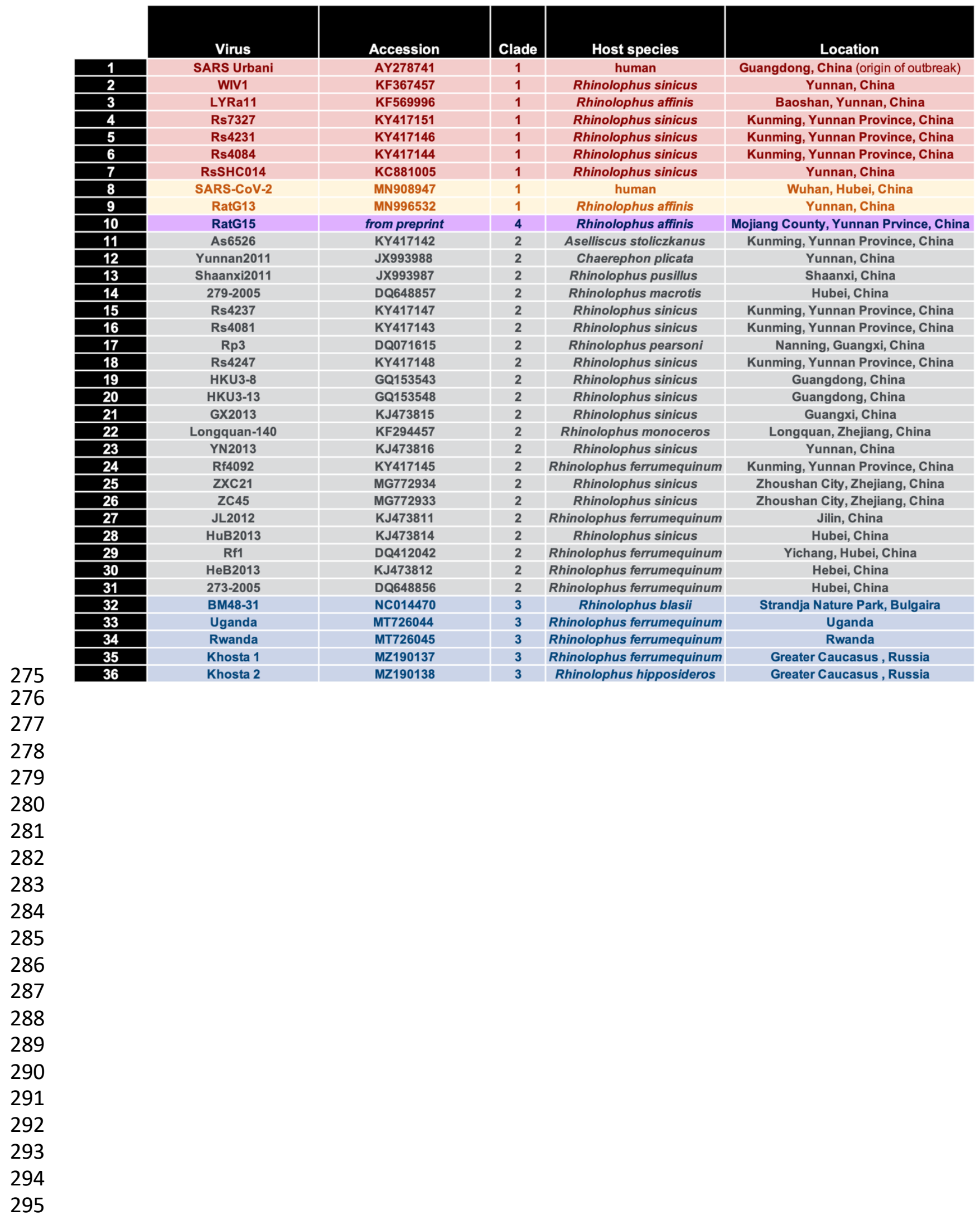




\section{REFERENCES}

1. Letko M, Marzi A, Munster V. Functional assessment of cell entry and receptor usage for SARS-CoV-2 and other lineage B betacoronaviruses. Nature Microbiology 2020; (4).

2. Hu B, Zeng LP, Yang XL, et al. Discovery of a rich gene pool of bat SARS-related coronaviruses provides new insights into the origin of SARS coronavirus. PLoS Pathog 2017; 13(11): e1006698.

302 3. Starr TN, Greaney AJ, Hilton SK, et al. Deep Mutational Scanning of SARS-CoV-2 Receptor 303 Binding Domain Reveals Constraints on Folding and ACE2 Binding. Cell 2020; 182(5): 1295-310 304 e20.

305 4. Guo H, Hu B, Si HR, et al. Identification of a novel lineage bat SARS-related coronaviruses 306 that use bat ACE2 receptor. Emerg Microbes Infect 2021; 10(1): 1507-14.

307 5. Alkhovsky S, Lenshin S, Romashin A, et al. SARS-like coronaviruses in horseshoe bats 308 (Rhinolophus spp.) in Russia, 2020. birXiv 2021.

309 6. Wells HL, Letko M, Ssebide B, et al. The evolutionary history of ACE2 usage within the subgenus Sarbecovirus. Virus Evol 2021.

311 7. Hoffmann M, Muller MA, Drexler JF, et al. Differential sensitivity of bat cells to infection

312 by enveloped RNA viruses: coronaviruses, paramyxoviruses, filoviruses, and influenza viruses.

313 PLoS One 2013; 8(8): e72942.

314 8. Shulla A, Heald-Sargent T, Subramanya G, Zhao J, Perlman S, Gallagher T. A transmembrane serine protease is linked to the severe acute respiratory syndrome coronavirus receptor and activates virus entry. J Virol 2011; 85(2): 873-82.

317 9. Peck KM, Cockrell AS, Yount BL, Scobey T, Baric RS, Heise MT. Glycosylation of mouse DPP4 plays a role in inhibiting Middle East respiratory syndrome coronavirus infection. $J$ Virol 2015; 89(8): 4696-9.

10. Wan Y, Shang J, Graham R, Baric RS, Li F. Receptor Recognition by the Novel Coronavirus from Wuhan: an Analysis Based on Decade-Long Structural Studies of SARS Coronavirus. J Virol 2020; 94(7).

323 11. Liu K, Tan S, Niu S, et al. Cross-species recognition of SARS-CoV-2 to bat ACE2. Proc Natl 324 Acad Sci U S A 2021; 118(1).

325 12. Ge XY, Li JL, Yang XL, et al. Isolation and characterization of a bat SARS-like coronavirus that uses the ACE2 receptor. Nature 2013; 503(7477): 535-8.

13. Menachery VD, Yount BL, Jr., Debbink K, et al. A SARS-like cluster of circulating bat coronaviruses shows potential for human emergence. Nat Med 2015; 21(12): 1508-13. 14. Yang XL, Hu B, Wang B, et al. Isolation and Characterization of a Novel Bat Coronavirus Closely Related to the Direct Progenitor of Severe Acute Respiratory Syndrome Coronavirus. J Virol 2015; 90(6): 3253-6. 15. Zhou P, Yang X, Wang $X$, et al. A pneumonia outbreak associated with a new coronavirus of probable bat origin. Nature 2020.

16. Drexler JF, Gloza-Rausch F, Glende J, et al. Genomic characterization of severe acute respiratory syndrome-related coronavirus in European bats and classification of coronaviruses based on partial RNA-dependent RNA polymerase gene sequences. J Virol 2010; 84(21): 1133649. 
340 18. Zhang Q, Zhang $\mathrm{H}$, Gao J, et al. A serological survey of SARS-CoV-2 in cat in Wuhan.

341 Emerg Microbes Infect 2020; 9(1): 2013-9.

342 19. Palmer MV, Martins M, Falkenberg S, et al. Susceptibility of white-tailed deer

343 (Odocoileus virginianus) to SARS-CoV-2. J Virol 2021.

344 20. Oude Munnink BB, Sikkema RS, Nieuwenhuijse DF, et al. Transmission of SARS-CoV-2 on 345 mink farms between humans and mink and back to humans. Science 2021; 371(6525): 172-7.

346 21. Fagre A, Lewis J, Eckley M, et al. SARS-CoV-2 infection, neuropathogenesis and transmission among deer mice: Implications for spillback to New World rodents. PLoS Pathog $348 \quad 2021 ; 17(5)$ : e1009585.

349 22. Di Guardo G. Susceptibility of white-tailed deer to SARS-CoV-2. Vet Rec 2021; 189(10):

350 408-9.

351 23. Chandler JC, Bevins SN, Ellis JW, et al. SARS-CoV-2 exposure in wild white-tailed deer 352 (Odocoileus virginianus). Proc Natl Acad Sci U S A 2021; 118(47).

353 24. Menachery VD, Dinnon KH, 3rd, Yount BL, Jr., et al. Trypsin treatment unlocks barrier for 354 zoonotic bat coronaviruses infection. J Virol 2019.

355 25. Zech F, Schniertshauer D, Jung C, et al. Spike residue 403 affects binding of coronavirus 356 spikes to human ACE2. Nat Commun 2021; 12(1): 6855.

357 26. Shang J, Ye G, Shi K, et al. Structural basis of receptor recognition by SARS-CoV-2. Nature 358 2020; 581(7807): 221-4.

359 27. Liu K, Pan X, Li L, et al. Binding and molecular basis of the bat coronavirus RaTG13 virus 360 to ACE2 in humans and other species. Cell 2021; 184(13): 3438-51 e10.

361 28. Letko M, Miazgowicz K, McMinn R, et al. Adaptive Evolution of MERS-CoV to Species 362 Variation in DPP4. Cell Rep 2018; 24(7): 1730-7.

363 29. Martinez DR, Schafer A, Leist SR, et al. Chimeric spike mRNA vaccines protect against 364 Sarbecovirus challenge in mice. Science 2021; 373(6558): 991-8.

365 30. Tortorici MA, Czudnochowski N, Starr TN, et al. Broad sarbecovirus neutralization by a 366 human monoclonal antibody. Nature 2021; 597(7874): 103-8.

367 31. Guindon S, Dufayard JF, Lefort V, Anisimova M, Hordijk W, Gascuel O. New algorithms 368 and methods to estimate maximum-likelihood phylogenies: assessing the performance of 369 PhyML 3.0. Syst Biol 2010; 59(3): 307-21.

$37032 . \quad$ Lefort V, Longueville JE, Gascuel O. SMS: Smart Model Selection in PhyML. Mol Biol Evol $371 \quad 2017 ; 34(9): 2422-4$. 